\title{
Secretome Analyses of Induced Pluripotent Stem Cells-Derived Neural Progenitors Cells from Patients with Schizophrenia and Controls
}

\author{
Danielle G. Junqueira*, Giuliana S. Zuccoli, Juliana M. Nascimento, Daniel Martins-de-Souza.
}

\begin{abstract}
Schizophrenia is a complex neuropsychiatric disorder, involving genetic and environmental factors. The disease results in a range of symptoms and it is related to changes during neurodevelopment. Therefore, the use of neural progenitor cells (NPCs) is an attractive model to better understand the molecular mechanisms from this disorder. We performed a proteomic analysis of the secretome obtained from NPC's culture media, obtained from patients with schizophrenia and mentally healthy controls. In our results, we found proteins associated to signaling process and cellular communication. And also identified proteins associated with transport mediated by vesicles, signal transduction, brain development, organization of the extracellular matrix and targeting proteins of the membrane. The study of the secretome obtained from NPCs allows the analysis of the proteins secreted singly and those coming vesicles such as exosomes. The analysis of the proteome from the culture medium will provide a better understanding of the content released from cultured cells and their communication as well as mechanisms potentially involved in the development of schizophrenia.
\end{abstract}

\section{Key words: \\ Secretome, Proteome, Schizophrenia}

\section{Introduction}

Schizophrenia is a complex psychiatric disorder, which is influenced by genetic and environmental factors, and it is characterized by positive, negative and cognitive symptoms, and also impairs the neurodevelopment ${ }^{1}$. Therefore, the use of neural progenitor cells (NPCs) is an interesting model to develop a better understanding of the mechanisms from this disorder ${ }^{2}$. Here we analyzed the secretome of NPCs in order to investigate the celullar communication and search for pathways related to the disorder.

The molecular aspects of the disease are not well understood and proteomics is one of the tools contributing on this regard. Proteomic analysis allows the identification of proteins and metabolic pathways which they are involved in ${ }^{3,4}$.

Here we aim to analyze the secretome from the cell culture medium of cells derived from patients with schizophrenia and mentally healthy controls, in order to identify possible proteins expressed differently associated to schizophrenia as well as a better understanding of the mechanisms of the disorder.

\section{Results and Discussion}

To perform secretome analyses we used media from induced pluripotent stem cells (iPSCs) from patients with schizophrenia and control cultures. The cells obtained from the patients and control were reprogrammed and differentiated in to neural lineages, resulting in neuron progenitor cells (NPCs). We colleted and concentrated the culture medium from NPCs, and then we executed the extraction and trypsin digestion of the proteins. The peptides obtained were subjected to state-of-the-art bottom up shotgun proteomics in a two dimensional liquid chromatography coupled to mass spectrometry in HDMSE mode. A label-free approach was used for proteome quantitation.
For the data analysis, we used softwares for the identification of the proteins obtained and for the association of the proteins with the metabolic pathways they operate in.

We quantified 1154 proteins, from which 362 were differentially expressed comparing patients with schizophrenia and control. We found proteins associated to signaling process and cellular communication. And also identified proteins associated with transport mediated by vesicles, signal transduction, brain development, organization of the extracellular matrix and targeting proteins of the membrane.

\section{Conclusions}

Data on NPCs secretome allows the analysis of the proteins secreted singly and those coming vesicles such as exosomes. The analysis of the proteome from the culture media provides a better understanding of the content released from the cultured cells and their communication as well as mechanisms involved in the development of schizophrenia.

\section{Acknowledgement}

This project was funded by FAPESP and CNPq.

${ }^{1}$ Kahn RS, Sommer IE, Murray RM, Meyer-Lindenberg A, Weinberger DR, Cannon TD, et al. Schizophrenia. Nat Rev Dis Prim [Internet]. 2015;(November):15067. Available from: http://www.nature.com/articles/nrdp201567

2 Brennand, K.J. et al., 2011. Modelling schizophrenia using human induced pluripotent stem cells. Nature, 473(7346), pp.221-225. Available at: http://www.nature.com/doifinder/10.1038/nature09915.

${ }^{3}$ Nascimento JM, Martins-de-Souza D. The proteome of schizophrenia. npj Schizophr [Internet]. 2015;1(October 2014):14003. Available from: http://www.nature.com/articles/npjschz20143

${ }^{4}$ Martins-de-Souza D. Proteomics as a tool for understanding schizophrenia. Clin Psychopharmacol Neurosci. 2011;9(3):95-101. 